\title{
Warm white light generating nanocrystal hybridized LEDs with high color rendering index
}

\author{
Hilmi Volkan Demir, Sedat Nizamoglu, Gulis Zengin \\ Department of Electrical and Electronics Engineering, Department of Physics, Nanotechnology Research Center, \\ Institute of Materials Science and Nanotechnology, Bilkent University, Ankara 06800 Turkey \\ Tel:[+90](312)2901021, e-mail: volkan@bilkent.edu.tr
}

Today lighting consumes $20 \%$ of the world electricity production [1]. Solid state lighting (SSL) is predicted to save $50 \%$ of the electricity consumption for lighting and consequently reduce carbon emission by 300 million tons per year [2]. Recently in SSL, nanocrystal (NC) based light emitting devices have made great progress [37]. Nanocrystals are particularly advantageous for use in white light sources because they feature tunable and relatively narrow emission across the visible spectral range. They further exhibit small overlap between their emission and absorption spectra, and also provide the ability to be easily and uniformly deposited in solid films using common techniques (spin casting, dip coating, etc.). However, despite their recent progress to date, such nanocrystal integrated white LEDs have not been demonstrated to exhibit warm correlated color temperatures (CCT) simultaneously with high color rendering indices (CRI) [3-7], though this is required for indoor lighting [1-3]. As a matter of fact, such high-CRI, warm-white LEDs have previously not been achieved using phosphors, which are the most commonly used luminophors, either [3].

In this talk to meet this demand, we will present our proposal, development, and demonstration of high-CRI, warm-white LEDs using custom-design nanocrystal luminophors. In our hybrid devices we used green and red emitting CdSe $/ \mathrm{ZnS}$ core-shell nanocrystals (with photoluminescence peak at $\lambda_{\mathrm{PL}}=555$ and $613 \mathrm{~nm}$, respectively) integrated on our blue InGaN/GaN light emitting diodes (with electroluminescence peak at $\lambda_{\mathrm{EL}}=452 \mathrm{~nm}$ ) [8]. Using red and green NCs on blue LED was important for efficient pumping of NCs and obtaining high CRI. In our designs using our nanocrystal emitters, the red emission above $650 \mathrm{~nm}$ was significantly avoided, unlike using phosphors that exhibit strong emission tail in the red above $650 \mathrm{~nm}$. As a result, in the case of using phosphors, the luminous efficacy (LE) of optical radiation (i.e., the ratio of the emitted luminous flux to the radiant flux) substantially drops (because the eye sensitivity function decreases quickly above $650 \mathrm{~nm}$ ). On the other hand, in our case, the use of these nanocrystal luminophors enabled to achieve white light generation with high LE in the warm-white region, while maintaining high CRI. Based on our careful designs and hybridization of the nanocrystal emitters, we developed and demonstrated three sets of proof-of-concept warm-white LEDs with high-quality white light properties as follows: 1.$)(\mathrm{x}, \mathrm{y})=(0.37,0.30), \mathrm{LE}=307 \mathrm{~lm} / \mathrm{W}$ of optical radiation, $\mathrm{CRI}=82.4, \mathrm{CCT}=3228 \mathrm{~K} ; 2.)(\mathrm{x}, \mathrm{y})=(0.38,0.31), \mathrm{LE}=323 \mathrm{~lm} / \mathrm{W}$ of optical radiation, $\mathrm{CRI}=81.0, \mathrm{CCT}=3190 \mathrm{~K}$; and 3. $)(x, y)=(0.37,0.30), \mathrm{LE}=303 \mathrm{~lm} / \mathrm{W}$ of optical radiation, $\mathrm{CRI}=79.6, \mathrm{CCT}=1982 \mathrm{~K}$, shown on $\mathrm{CIE}$ chromaticity diagram in Fig. 1 [8].

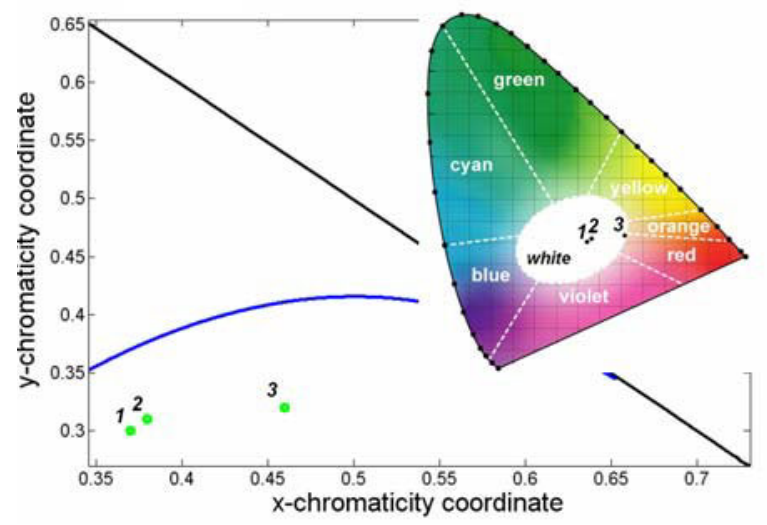

Figure 1.Tristimulus coordinates of our nanocrystal-hybridized warm-white LEDs (Samples 1, 2 and 3 shown in green points 1 , 2 and 3) along with the planckian locus (shown in blue line). The inset shows these tristimulus coordinates on a CIE 1931 chromaticity diagram.

For the first warm-white LED sample, we hybridized $0.22 \mathrm{mg}(0.578 \mathrm{nmol})$ of red-emitting CdSe $/ \mathrm{ZnS}$ core-shell NCs and subsequently $0.26 \mathrm{mg}(2.166 \mathrm{nmol})$ of green-emitting NCs on blue LED $\left(\lambda_{\mathrm{EL}}=452 \mathrm{~nm}\right)$. These nanocrystals were designed to have diameters of $9.6 \mathrm{~nm}$ and $7.7 \mathrm{~nm}$ (with a size distribution of $\pm 5 \%$ ) to emit at the peak wavelengths of $613 \mathrm{~nm}$ and $555 \mathrm{~nm}$, respectively. Red emitting nanocrystals increased the color temperature of the resulting hybrid device; but, at the same time they may have undesirably reduced the luminous efficacy by contributing to emission above $650 \mathrm{~nm}$. Therefore, it was necessary to carefully set the 
emission wavelength and amount of the red NCs. In addition, the green emitting nanocrystals balanced out the red emission at $555 \mathrm{~nm}$ along with the blue LED emission at $452 \mathrm{~nm}$ by holding the tristimulus coordinates in the white region and the color rendering index sufficiently high. We obtained the luminescence of this hybrid LED at various current injection levels at room temperature as shown in Fig. 2 (bottom), leading to the photometric properties of $(\mathrm{x}, \mathrm{y})=(0.37,0.30), \mathrm{LE}=307 \mathrm{~lm} / \mathrm{W}$ of optical radiation, $\mathrm{CRI}=82.4$, and $\mathrm{CCT}=3228 \mathrm{~K}$. Such a hybrid white LED satisfied the future SSL criterion of CRI $\sim 80$ with a warm CCT.

For the second warm-white LED sample, we integrated $0.13 \mathrm{mg}(1.083 \mathrm{nmol}) \mathrm{CdSe} / \mathrm{ZnS}$ core-shell NCs emitting at $555 \mathrm{~nm}$ in green region and then $0.44 \mathrm{mg}(1.156 \mathrm{nmol})$ of NCs emitting at $613 \mathrm{~nm}$ in red region on blue LED $\left(\lambda_{\mathrm{EL}}=452 \mathrm{~nm}\right)$. The luminescence of this hybrid LED at various current injection levels is depicted in Fig. 2 (middle). This implementation experimentally led to $(\mathrm{x}, \mathrm{y})=(0.38,0.31), \mathrm{LE}=323 \mathrm{~lm} / \mathrm{W}$ of optical radiation, $\mathrm{CRI}=81.0$, and $\mathrm{CCT}=3190 \mathrm{~K}$. In this case, the correlated color temperature became warmer and decreased to $3190 \mathrm{~K}$ because of the increased relative intensity of the red-emitting nanocrystals. Therefore, this light source generated warmer white light, while keeping its chromaticity operating point in the white at the same time. Also, the luminous efficacy of its emitted spectrum reached a relatively high level of $323 \mathrm{~lm} / \mathrm{W}$. Though its color rendering index decreased to 81.0, this light source still satisfied the criterion for the future indoor SSL sources.

As the last demonstration of our hybrid warm-white LEDs, we designed to hybridize $0.13 \mathrm{mg}(1.083 \mathrm{nmol})$ of green-emitting NCs $\left(\lambda_{\mathrm{PL}}=555 \mathrm{~nm}\right)$ and $0.66 \mathrm{mg}(1.734 \mathrm{nmol})$ of red-emitting NCs $\left(\lambda_{\mathrm{PL}}=613 \mathrm{~nm}\right)$ on blue LED $\left(\lambda_{\mathrm{EL}}=452 \mathrm{~nm}\right)$. The resulting spectra of hybrid LED at various levels of current injection are presented in Fig. 2 (top), corresponding to $(\mathrm{x}, \mathrm{y})=(0.37,0.30), \mathrm{LE}=303 \mathrm{~lm} / \mathrm{W}$ of optical radiation, $\mathrm{CRI}=79.6$, and $\mathrm{CCT}=1982 \mathrm{~K}$. In this design, the operating point was located approximately on the boundary of white region near to the red-color end as shown in Fig. 1. Therefore, this hybrid white LED generated highly warm-white light at an extra-low correlated color temperature of $1982 \mathrm{~K}$.

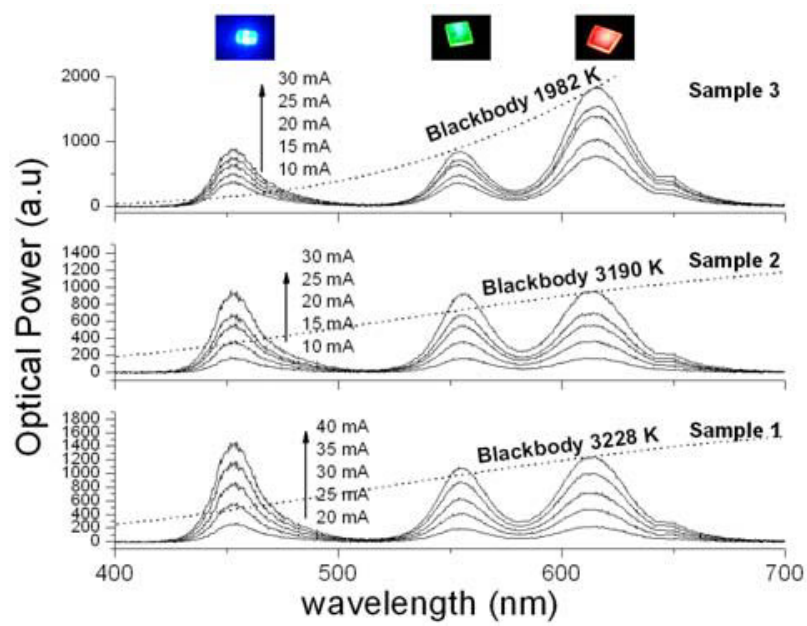

Figure 2. Luminescence spectra of our nanocrystal hybridized warm-white LEDs (Samples 1-3) when electrically driven at room temperature.

In conclusion, hybridizing custom-design $\mathrm{CdSe} / \mathrm{ZnS}$ core-shell $\mathrm{NC}$ emitters on InGaN/GaN based blue LEDs, we demonstrated three warm-white light sources with desirably low CCT ranging from $3227 \mathrm{~K}$ to $1982 \mathrm{~K}$ as is required for SSL indoor applications. In these proof-of-concept demonstrations, high color rendering indices (82.4) and high luminous efficacies of emitted spectra $(327 \mathrm{~lm} / \mathrm{W})$ were achieved, while the color temperature was simultaneously kept low as desired. Our proof-of-concept demonstrations indicated that such nanocrystal luminophor based warm-white LEDs with high-quality photometric properties hold great promise especially for future indoor lighting applications.

This work is supported by EU PHOREMOST Network of Excellence 511616, EU Marie Curie IRG MOON 021391, TUBITAK EEEAG No. 106E020, 104E114, 107E088, 107E297, 105E065, and 105E066. HVD also acknowledges ESF-EURYI and TUBA-GEBIP.

Reference

[1] http://www.netl.doe.gov/ss1/PDFs/oida_led-oled_rpt.pdf, 2008.

[2] http://lighting.sandia.gov, 2008.

[3] E. F. Schubert, Cambridge University Press, 2006.

[4] H. Chen et al, IEEE Photon. Tech. Lett., 18, 1430, 2006.

[5] S. Nizamoglu et al., Nanotechnology, 18, 065709, 2007.

[6] M. Ali et al., Nanotechnology, 18, 075401, 2007.

[7] H. V. Demir et al., New J. of Phys., 9, 362, 2007.

[8] S. Nizamoglu et al., Appl. Phys. Lett., 92, 031102, 2008. 